\title{
ARTIGO ORIGINAL Distorções do pensamento em pacientes deprimidos: frequência e tipos
}

\author{
Distortions of thinking in depressed patients: frequency and type \\ Heydrich Lopes Virgulino de Medeiros'1, Everton Botelho Sougey²
}

\section{Palavras-chave}

Depressão, distorções do pensamento, desesperança, distorções cognitivas.

\section{Keywords}

Depression, thought distortions, hopelessness, cognitive distortions.

\section{RESUMO}

Objetivo: O objetivo deste estudo foi identificar as principais distorções do pensamento (DP) em pacientes deprimidos, sua frequência, bem como se há relação delas com dados clínicos e sociodemográficos. Método: Estudo descritivo de corte transversal com 75 voluntários entre 18 e 65 anos, residentes na cidade de João Pessoa/PB. Aplicaram-se a escala SCID - I, apenas a parte dedicada ao diagnóstico de depressão, o Inventário de Beck para Depressão (IBD) e questionários para avaliação de dados clínicos e sociodemográficos. Foram pesquisadas as principais distorções do pensamento descritas na literatura. Resultados: As distorções do pensamento mais prevalentes foram desesperança (69,3\%), hipocondria (62,7\%), ideias de fracasso $(61,3 \%)$ e desejo de morte $(60,0 \%)$. As DP ideias de culpa, desejo de morte e ideias suicidas foram as únicas a ocorrerem exclusivamente em pacientes com IBD > 10. Apenas 13,3\% dos pacientes não apresentaram no momento da entrevista nenhuma distorção do pensamento. Houve associação estatisticamente significativa entre várias DP e variáveis clínicas e sociodemográficas $(p<0,05)$. Conclusão: Conclui-se que as distorções do pensamento são altamente prevalentes em indivíduos deprimidos, merecendo maior investigação por parte dos profissionais de saúde mental envolvidos no atendimento a pacientes deprimidos.

\section{ABSTRACT}

Objective: This study aimed to identify the main thought distortions (TD) in depressed patients, their frequency as well as if there is any relation of such distortions with the clinical and sociodemographical data. Method: Descriptive study of transversal cut involving 75 volunteers between 18 and 65 years old, dwellers of João Pessoa/PB. The SCID-I scale was used, but just the part devoted to depression diagnosis, the Beck Depression Inventory (BDI) and questionnaires for evaluating the clinical and socio-demographical data. The main thought distortions, described in the literature, were researched. Results: The most prevalent thought distortions were hopelessness (69,3\%), hypochondria (62,7\%), failure ideas (61,3\%) and death desire (60,0\%). The thought distortions (TD) referring to guilt ideas, death desire and suicidal ideas were the only ones which occurred in patients with IBD > 10. Only 13,3\% of the patients did not present, at the moment of the interview, any thought distortion. There was a statistically meaningful association among several TDs and clinical and socio-demographical variables $(p<0,05)$. Conclusion: We conclude that thought distortions are highly prevalent in depressed individuals, deserving thus, a major investigation of these by the mental health professionals involved in taking care of depressed patients.

1 Universidade Federal de Campina Grande (UFCG).

2 Universidade Federal de Pernambuco (UFPE), Departamento de Neuropsiquiatria, Programa de Pós-graduação em Neuropsiquiatria e Ciências do Comportamento

Recebido em

Aprovado em

9/10/2010
Endereço para correspondência: Heydrich Lopes Virgulino de Medeiros Av. Manoel Morais, 320, Manaíra - 50308-230 - João Pessoa, PB E-mail: hvirgulino@hotmail.com 


\section{INTRODUÇÃO}

O transtorno depressivo maior (TDM) é uma doença grave, descrita desde a Antiguidade e que acomete o indivíduo geralmente entre a terceira e a quarta década de vida, cursando com prejuízos sociais e financeiros, além de promover aumento da morbimortalidade e danos físicos. Apresenta alta prevalência ao longo da vida, variando de 5,1\% a 15,7\%, com as muIheres sendo duas vezes mais acometidas quando comparadas com os homens ${ }^{1}$, tendo ainda caráter crônico e recorrente em $75 \%$ dos casos². Além disso, a depressão é mencionada como a quarta maior causa de incapacitação no mundo, com projeção para ser o principal motivo de incapacitação em $2020^{3}$.

Dentre os sintomas relacionados ao quadro depressivo, os sintomas cognitivos (SC) têm ganhado interesse gradativo dos pesquisadores nos últimos anos, especialmente por estarem presentes mesmo estando os indivíduos eutímicos ${ }^{4}$ e por terem relação muito próxima com a evolução clínica da doença e seu prognóstico. Segundo Sougey e Petribu ${ }^{5}$, os SC podem ainda ser subdivididos em dois grupos: o que diz respeito ao rendimento e o que se refere ao julgamento. Esses padrões de pensamentos presentes na depressão carecem ainda, na literatura, de terminologia consensual. A descrição psicopatológica situa-se nas alterações do conteúdo do pensamento 6 . Todavia, expressões como "pensamentos catatímicos", "pensamentos disfuncionais", "erros de julgamento", "distorções cognitivas", "pensamentos deliroides", "distorções do julgamento", entre outras possíveis, poderiam ser utilizadas para designar o objeto de nosso interesse neste estudo. Adotou-se, então, o termo distorções do pensamento (DP) como a mais próxima denominação para as alterações não delirantes da interpretação e atribuição de significados às vivências e aos fenômenos da existência.

Há relatos de que as DP estão presentes em $67 \%$ dos pacientes deprimidos ${ }^{7}$. Trata-se de ideias de culpa, pensamentos de autodepreciação, desesperança (pessimismo), ideias de fracasso, pensamentos de autocompaixão, ideias suicidas, hipocondria, pensamentos e desejo de morte, entre outros. Segundo o modelo cognitivo, humor e comportamentos negativos seriam usualmente resultados de pensamentos e crenças distorcidas, interferindo tanto no surgimento quanto na manutenção da depressão ${ }^{8}$. Esses sintomas compõem 38\% dos itens do Inventário de Beck para Depressão (IBD) e cerca de 20\% e 28\% dos sintomas das escalas de Montgomery e Hamilton, respectivamente. Além disso, alguns sintomas guardam importância por si, como a ideação suicida, e outros, como desesperança e ideias de culpa, possuem forte fator preditivo para o desenvolvimento da ideação suicida ${ }^{10,11}$.

Considerando a importância clínica dessa dimensão da sintomatologia depressiva, o presente estudo tem por objetivo identificar as principais DP em pacientes acometidos de depressão, sua frequência e possíveis associações com variáveis sociodemográficas e clínicas.

\section{MÉTODO}

\section{Desenho do estudo}

Trata-se de um estudo descritivo do tipo corte transversal.

\section{Amostra}

Foi considerada uma amostra aleatória ou casual simples de $n=75$ pacientes regularmente atendidos no Centro de Atenção Psicossocial (CAPS), no Ambulatório de Psiquiatria do Hospital Psiquiátrico Juliano Moreira e no Ambulatório de Psiquiatria do Centro Atenção Básica em Saúde (CAIS) de Cruz das Armas, todos situados na cidade de João Pessoa/ PB. Os critérios de inclusão foram ter entre 18 e 65 anos e diagnóstico de transtorno depressivo maior (TDM), pelo Manual de Diagnóstico e Estatística dos Transtornos Mentais, $4^{a}$ edição revisada (DSM-IV-TR), e residir na cidade de João Pessoa. Os critérios de exclusão foram: ser acometido de doença orgânico-cerebral ou se recusar, a qualquer momento, a assinar o termo de consentimento livre e esclarecido (TCLE). O projeto foi aprovado pelo Comitê de Ética em Pesquisa da Secretaria de Estado da Saúde da Paraíba. A coleta de dados ocorreu entre fevereiro/2008 e outubro/2008.

\section{Procedimentos}

Após serem esclarecidos sobre os métodos da pesquisa, os que concordaram em participar (100\%) assinaram o TCLE. Nenhum paciente solicitou retirada da assinatura do TCLE em qualquer fase da pesquisa. Após assinatura, foi aplicada a SCID-I como padrão-ouro para confirmação diagnóstica ${ }^{12}$. Em sequência, os pacientes responderam a perguntas relativas aos questionários sociodemográficos e de características clínicas e, de forma autoaplicável, ao IBD.

Após a aplicação do IBD, os pacientes foram separados em dois grupos: IBD > 10 (não eutímicos) e IBD $<10$ (eutímicos). Houve pesquisa da associação das DP e dados sociodemográficos e clínicos apenas nos voluntários com IBD > 10 .

O IBD foi utilizado para verificar a intensidade do episódio depressivo, bem como para obter sete das nove DP pesquisadas, correspondendo aos itens do IBD na seguinte ordem: item 2 (desesperança), 3 (ideias de fracasso) 5 (ideias de culpa), 7 (pensamentos de autocompaixão), 8 (pensamentos de autodepreciação), 9 (ideias suicidas) e 20 (hipocondria) ${ }^{13}$. As DP "desejo de morte" e "pensamentos de morte" foram pesquisadas por meio de perguntas feitas diretamente aos pacientes, como segue: "Na última semana o(a) senhor(a) tem pensado muito na morte, seja na morte de pessoas vivas ou já mortas, ou mesmo na sua própria morte? Esse tem sido um tema recorrente, pensar na morte?", para o pensamento de morte; e "Na última semana, o(a) senhor(a) tem desejado estar morto, desaparecer, mas sem que o(a) senhor(a) cause sua própria morte?", para o desejo de morte. 


\section{Análise estatística}

Após a construção de um banco de dados, as informações foram transferidas para o pacote estatístico SPSS (Statistical Package for Social Sciences), versão 13.0.

Na sequência, e de acordo com objetivos específicos propostos, foram utilizadas técnicas da estatística inferencial bivariada e multivariada, com uso do pacote estatístico SPSS, versão 13.0, no nível de 5\% de significância.

\section{RESULTADOS}

Setenta e cinco pacientes participaram da pesquisa. A tabela 1 mostra que a idade média da amostra foi de 42,7 anos, com desvio-padrão de 12,2 anos. Houve considerável diferença entre os gêneros, com predominância do sexo feminino $(82,7 \% \times 17,3 \%)$. Houve também predomínio de pacientes casados (45,3\%). A tabela 2 evidencia que a idade média em que os pacientes tiveram o primeiro episódio foi aos 36 anos, com desvio-padrão de 12,6 anos. Quase metade (49,3\%) teve quatro ou mais episódios ao longo da vida. A maioria dos pacientes nunca necessitou de internamento hospitalar decorrente de algum episódio depressivo (82,7\%) e 57,3\% em algum momento referiram ter tentado suicidar-se.

Tabela 1. Perfil sociodemográfico dos pacientes deprimidos pesquisados $(n=75)$

\begin{tabular}{|c|c|c|c|}
\hline \multirow[b]{2}{*}{ Variável } & \multirow[b]{2}{*}{ Categoria } & \multicolumn{2}{|c|}{ Pacientes pesquisados } \\
\hline & & $N^{0}$ de pacientes $(n)$ & Percentual (\%) \\
\hline \multirow[t]{8}{*}{ Idade (anos) } & 18 a 25 & 6 & 8,0 \\
\hline & 26 a 33 & 13 & 17,3 \\
\hline & 34 a 41 & 13 & 17,3 \\
\hline & 42 a 49 & 16 & 21,3 \\
\hline & 50 a 57 & 19 & 25,3 \\
\hline & 58 a 65 & 8 & 10,7 \\
\hline & $\begin{array}{l}\text { Idade média }= \\
42,7 \text { anos }\end{array}$ & Mínimo = 18 & \\
\hline & $\begin{array}{l}\text { Desvio-padrão }= \\
12,2 \text { anos }\end{array}$ & Máximo $=65$ & \\
\hline \multirow[t]{2}{*}{ Gênero } & Feminino & 62 & 82,7 \\
\hline & Masculino & 13 & 17,3 \\
\hline \multirow[t]{3}{*}{ Cor } & Parda & 48 & 64,0 \\
\hline & Negra & 19 & 25,3 \\
\hline & Branca & 8 & 10,7 \\
\hline \multirow[t]{5}{*}{ Estado civil } & Solteiro(a) & 19 & 25,3 \\
\hline & Casado(a) & 34 & 45,3 \\
\hline & Divorciado(a) & 14 & 18,7 \\
\hline & Viúvo(a) & 4 & 5,3 \\
\hline & União consensual & 4 & 5,3 \\
\hline \multirow[t]{4}{*}{ Grau de escolaridade } & Não alfabetizado & 3 & 4,0 \\
\hline & Fundamental & 36 & 48,0 \\
\hline & Ensino Médio & 24 & 32,0 \\
\hline & Superior & 12 & 16,0 \\
\hline \multirow[t]{2}{*}{ Situação empregatícia } & Empregado(a) & 36 & 48,0 \\
\hline & Desempregado(a) & 39 & 52,0 \\
\hline Total & & 75 & 100,0 \\
\hline
\end{tabular}

Tabela 2. Características clínicas dos pacientes da amostra

\begin{tabular}{|c|c|c|c|}
\hline Variável & Categoria & $\mathrm{N}^{0}$ de pacientes (n) & Percentual (\%) \\
\hline $\begin{array}{l}\text { Idade do } 1^{0} \text { episódio } \\
\text { depressivo }\end{array}$ & $\begin{array}{l}\text { Idade média }= \\
36,0 \text { anos } \\
\text { Desvio-padrão }= \\
12,6 \text { anos }\end{array}$ & $\begin{array}{l}\text { Mínimo = } \\
14 \text { anos } \\
\text { Máximo = } \\
63 \text { anos }\end{array}$ & $\begin{array}{l}\text { Idade mediana } \\
=35\end{array}$ \\
\hline Número de episódios & $\begin{array}{l}1 \\
2 \text { a } 3 \\
4 \text { ou mais }\end{array}$ & $\begin{array}{l}17 \\
21 \\
37\end{array}$ & $\begin{array}{l}22,7 \\
28 \\
49,3\end{array}$ \\
\hline $\begin{array}{l}\text { № de internamentos em } \\
\text { hospital psiquiátrico ou clínico }\end{array}$ & $\begin{array}{l}\text { Nenhum } \\
1 \\
2 \text { a } 3 \\
4 \text { ou mais }\end{array}$ & $\begin{array}{r}62 \\
7 \\
2 \\
4\end{array}$ & $\begin{array}{r}82,7 \\
9,3 \\
2,7 \\
5,3\end{array}$ \\
\hline № de tentativas de suicídio & $\begin{array}{l}\text { Nenhuma } \\
1 \\
2 \text { a } 3 \\
4 \text { ou mais }\end{array}$ & $\begin{array}{r}43 \\
12 \\
12 \\
8\end{array}$ & $\begin{array}{l}57,3 \\
16,0 \\
16,0 \\
10,7\end{array}$ \\
\hline Total & & 75 & 100,0 \\
\hline
\end{tabular}

A figura 1 mostra a frequência e a distribuição das DP em toda a amostra, com destaque para a desesperança, que foi o pensamento mais prevalente (69,3\%). Ao se analisarem apenas os pacientes com depressão clínica (IBD > 10), as proporções em geral aumentam consideravelmente, como pode ser observado na tabela 3, chegando a DP desesperança a atingir a frequência de 85,5\%.

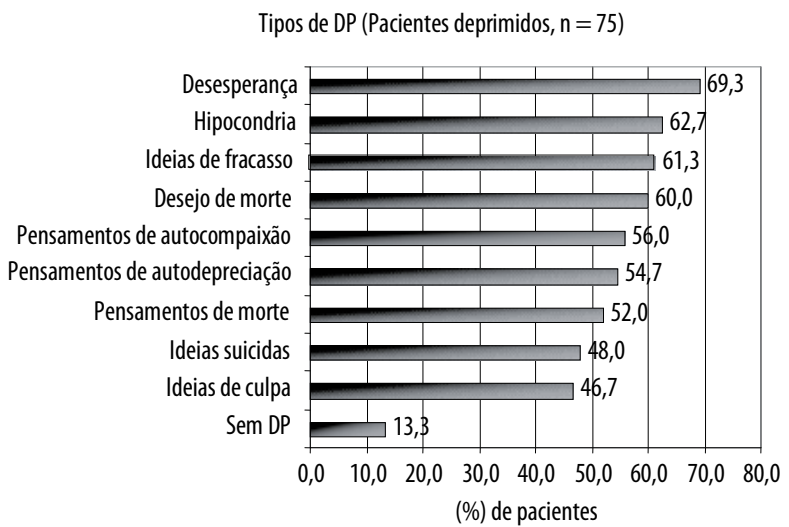

Figura 1. Tipos de DP indicadas pelos pacientes deprimidos sem sintomas psicóticos.

A presença da DP hipocondria está associada significativamente com o nível de escolaridade dos pacientes deprimidos, apresentando maior frequência para escolaridade mais baixa, Fundamental, quando comparada à categoria de referência: Superior $(88,9 \% \times 36,4 \%$, sig. $p$ - valor $=0,011<0,05$, $\mathrm{OR}=0,07$ e IC $[0,01 ; 0,40])$.

As demais variáveis do perfil sociodemográfico e todas referentes às características clínicas não apresentaram diferenças significativas em relação à categoria de referência considerada, $\mathrm{p}$ - valor $>0,05$, respectivamente.

A DP desejo de morte está associada significativamente com o estado civil dos pacientes deprimidos, apresentando maior frequência para a categoria de não casados, quando comparada à categoria de referência casados $(70,0 \% \times 96,0 \%$, sig. $\mathrm{p}$ - valor $=0,033<0,05, \mathrm{OR}=10,29$ e IC $[1,20 ; 88,07])$. 
Tabela 3. Frequência das DP segundo situação da depressão (IBD)

\begin{tabular}{|c|c|c|c|c|c|c|}
\hline \multirow{3}{*}{ DP-Distorções do pensamento } & \multicolumn{4}{|c|}{ IBD - Intensidade da depressão } & \multicolumn{2}{|c|}{ Teste de associação ${ }^{\mathrm{a}}$} \\
\hline & \multicolumn{2}{|c|}{ Depressão (-) } & \multicolumn{2}{|c|}{ Depressão(t) } & \multirow{2}{*}{$\begin{array}{c}\text { Estatística } \\
\chi^{2}\end{array}$} & \multirow{2}{*}{$\begin{array}{c}\text { Signif. } \\
\text { p-valor* }\end{array}$} \\
\hline & $n$ & $\%$ & $n$ & $\%$ & & \\
\hline 1. Desesperança & 5 & 25,0 & 47 & 85,5 & 25,210 & 0,000 \\
\hline 2. Hipocondria & 7 & 35,0 & 39 & 72,7 & 8,923 & 0,006 \\
\hline 3. Ideias de fracasso & 2 & 10,0 & 44 & 80,0 & 30,304 & 0,000 \\
\hline 4. Desejo de morte & - & - & 45 & 81,8 & - & - \\
\hline 5. Pensamento de autocompaixão & 2 & 10,0 & 40 & 72,7 & 23,481 & 0,000 \\
\hline 6. Pensamento de autodepreciação & 1 & 5,0 & 40 & 72,7 & 27,147 & 0,000 \\
\hline 7. Pensamentos de morte & 3 & 15,0 & 36 & 65,5 & 14,958 & 0,000 \\
\hline 8. Ideias suicidas & - & - & 36 & 65,5 & - & - \\
\hline 9. Ideias de culpa & - & - & 35 & 63,6 & - & - \\
\hline 10. Sem DP & 10 & 50,0 & - & - & - & - \\
\hline Total & 20 & 100,0 & 55 & 100,0 & - & - \\
\hline
\end{tabular}

a Teste de associação de qui-quadrado.

* Resultado significativo, $p$ - valor $<0,01$.

As demais variáveis do perfil sociodemográfico e todas referentes às características clínicas também não apresentaram diferenças significativas em relação à categoria de referência considerada, $p$ - valor $>0,05$, respectivamente.

Já a DP pensamentos de autocompaixão foi associada significativamente com a faixa etária dos pacientes deprimidos, diminuindo a frequência com o aumento da idade, quando comparada à categoria de referência 50 a 65 anos $(92,3 \% \mathrm{x}$ $44,4 \%$, sig. $p$ - valor $=0,018<0,05, O R=0,07$ e IC $[0,01 ; 0,63])$, além de também ter mostrado relevância estatística quando comparada com a característica clínica idade do 10 episódio, apresentando menor frequência nas idades mais avançadas, categoria de referência acima de 35 anos $(87,5 \% \times 52,2 \%$, sig. $p$ - valor $=0,002<0,05, O R=6,42$ e IC $[1,70 ; 24,24])$.

A DP pensamentos de autodepreciação está associada significativamente com a faixa etária dos indivíduos deprimidos, diminuindo a frequência com o aumento da idade, quando comparada à categoria de referência 50 a 65 anos $(84,6 \% \times 44,4 \%$, sig. $p$ - valor $=0,003<0,05, \mathrm{OR}=6,88 \mathrm{e} \mathrm{IC}$ $[1,17 ; 40,38])$. Não houve associação estatisticamente significante com as demais variáveis.

Dentre todas as variáveis, a ideação suicida foi associada significativamente apenas com o estado civil dos pacientes, apresentando maior prevalência para a categoria de não casados, quando comparada à categoria de referência casados $(53,3 \% \times 80,0 \%$, sig. $p$ - valor $=0,043<0,05, O R=3,50$ e IC $[1,04 ; 11,79])$.

A DP ideias de culpa está associada significativamente com a faixa etária dos pacientes deprimidos, diminuindo a frequência com o aumento da idade, quando comparada à categoria de referência 18 a 33 anos $(84,6 \% \times 44,4 \%$, sig. $p$ - valor $=0,042<0,05, O R=0,26$ e IC $[0,10 ; 0,96])$. Houve associação também entre a presença da ideia de culpa com o nível de escolaridade dos indivíduos deprimidos, apresentando menor prevalência para escolaridade mais baixa, Fundamental, quando comparada à categoria de referência
Superior $(81,8 \% \times 37,0 \%$, sig. $\mathrm{p}-$ valor $=0,003<0,05, \mathrm{OR}=0,04$ e IC $[0,00 ; 0,32])$.

Os pensamentos desesperança, ideias de fracasso e pensamentos de morte não mostraram associação estatisticamente significante com nenhuma das variáveis sociodemográficas e clínicas pesquisadas.

\section{DISCUSSÃO}

Um dos consensos na literatura acerca da depressão é o fato de essa enfermidade acometer duas vezes mais mulheres em relação aos homens ${ }^{14,15}$.

No presente estudo, houve significativa diferença entre os gêneros, na ordem de 5:1, o que pode ser justificado, além da esperada maior prevalência no gênero feminino, pelo já conhecido fato de que as mulheres costumam procurar mais os serviços de saúde. A idade média de 42,7 anos para as pessoas acometidas de depressão (quinta década) é um achado que pode refletir as dificuldades da população local em ter acesso à saúde especializada, visto que durante o período de coleta todo o município era contemplado com apenas dois ambulatórios de psiquiatria. Tal dificuldade de acesso ao psiquiatra, acredita-se, propicia que pacientes passem anos oscilando entre períodos de recidiva e remissão de sintomas, retardando o início do tratamento. Esse fator, associado ao achado do primeiro episódio ter ocorrido em média aos 36 anos, tardio segundo a literatura ${ }^{16}$, pode justificar a média de idade alta da população pesquisada. Indivíduos com dois ou mais episódios somaram 77,3\% da amostra, corroborando o conceito de que a depressão é uma doença de caráter recorrente ${ }^{17}$.

No que concerne à tentativa de suicídio, apesar de 42,7\% dos indivíduos terem citado ao menos uma tentativa de suicídio ao longo da história da doença, apenas 17,3\% afirmaram terem necessitado de internamento hospitalar (clínico ou psiquiátrico) em algum momento. Tal achado pode ser 
justificado por duas características que podem estar presentes nas tentativas de suicídio: baixa intencionalidade e impulsividade ${ }^{18,19}$, não levando, em todos os casos, ao internamento hospitalar.

Nos raros estudos encontrados, em que essas DP foram especificamente pesquisadas ${ }^{10,11}$, a desesperança mostrou forte associação com o surgimento da ideação suicida. No entanto, neste estudo, apesar de ser o pensamento distorcido mais prevalente, não houve associação estatisticamente significativa entre desesperança e dados sociodemográficos e clínicos, o que pode ser justificado pela provável distribuição uniforme dessa DP, considerando sua grande frequência entre os voluntários com IBD > 10 (85,5\%). O mesmo comportamento ocorreu com ideia de fracasso e pensamento de morte.

Em estudo realizado em três grandes centros urbanos ${ }^{20}$, a hipocondria foi o sintoma depressivo mais prevalente, com taxa de $15 \%$. A alta prevalência da DP hipocondria $(72,7 \%)$ nos voluntários com IBD > 10 só reforça a importância e frequência com que esse padrão de pensamento ocorre. Houve associação desse sintoma com o nível de escolaridade, sendo mais frequente quanto menor a escolaridade. Apesar de não ter sido identificado na literatura nenhum estudo que associasse o sintoma hipocondria com pouca escolaridade, vários trabalhos ${ }^{21-23}$ apontam a pouca escolaridade como importante fator de risco para o desenvolvimento de depressão. Sendo a hipocondria um dos sintomas depressivos mais comuns, como já citado, natural o resultado que mostra sua associação de forma inversamente proporcional ao grau de escolaridade.

O sintoma ideia suicida e o desejo de morte mostraram associação estatisticamente significante entre os indivíduos não casados (solteiros, viúvos e separados) quando comparados com os casados. No que se refere à ideia suicida, tal achado corrobora a literatura acerca do tema ${ }^{24}$, visto que estar casado é considerado fator protetor para o suicídio. Apesar da escassez de estudos, acredita-se que o mesmo raciocínio pode ser usado para desejo de morte, cuja diferença básica para ideação suicida está no agente causador da morte, mantendo-se em comum o desejo de dar fim à própria vida.

As ideias de culpa têm sua frequência aumentada de forma inversamente proporcional à idade e diretamente proporcional à escolaridade. Estudo comparando diferenças nos padrões de sintomas depressivos entre pacientes jovens e mais velhos evidenciou que os jovens tendem mais a desenvolver dentro do quadro depressivo a ideia de culpa ${ }^{25}$. Não se encontrou na literatura qualquer associação entre ideia de culpa e maior grau de escolaridade.

A tríade cognitiva ${ }^{26}$ compõe a visão negativa de si, do mundo e do futuro. Relevante o achado de que as DP relacionadas com a visão de si mesmo (ideia de culpa e pensamentos de autodepreciação e de autocompaixão), com exceção da ideia de fracasso, mostraram associação com baixa faixa etária. Em estudo conduzido por Balsis e Cully ${ }^{27}$, evidenciou-se que pacientes mais velhos, quando do preenchimento de escalas ou no fornecimento de dados para anamnese, tendem a endossar menos a presença de sintomas depressivos relacionados à cognição e ao conteúdo do pensamento e a valorizar mais os sintomas físicos. Desse modo, pacientes mais jovens tendem a dar mais ênfase a esses sintomas.

Apenas as distorções desejo de morte, ideias suicidas e ideias de culpa ocorreram exclusivamente em indivíduos com IBD > 10, o que pode sugerir forte fator de especificidade entre esses sintomas e a depressão. Em contrapartida, alguns desses pensamentos estiveram presentes mesmo em indivíduos considerados eutímicos (IBD < 10), como foi o caso da hipocondria, presente em 35\% dos voluntários eutímicos. Essa alta frequência pode ser justificada, à parte o quadro depressivo, pela presença de somatizações. Estas podem estar presentes em $60 \%$ dos pacientes acometidos de $\mathrm{TDM}^{28}$, dificultando a interpretação desse achado como pertencente genuinamente ao quadro depressivo ou a uma comorbidade com transtorno de somatização.

Desconhece-se qualquer trabalho que tenha pesquisado a frequência das ideias de fracasso e desejo de morte, DP muito frequentes em nossa amostra. Todavia, chama atenção a ausência de associação das DP com a variável tentativa de suicídio. Deve-se ressaltar que a pesquisa não foi realizada em campo aberto, mas sim em serviços especializados, nos quais há uma tendência natural pela procura de atendimento por pacientes acometidos de sintomas.

A alta prevalência de alguns sintomas pode ter sido causada por algum transtorno de personalidade enquanto condição comórbida, visto que a instabilidade emocional presente em alguns desses transtornos ${ }^{29}$ pode ter relação com DP como desesperança, ideias suicidas, pensamentos de morte etc., o que evidencia uma limitação do estudo, pois este poderia ter melhor acurácia caso se usasse como critério de exclusão ser acometido de transtorno de personalidade. Para isso, haveria necessidade de aplicação da SCID-II, o que não foi contemplado na metodologia.

\section{CONCLUSÃO}

Apesar das dificuldades quanto à delimitação do conceito, conclui-se que a definição psicopatológica que melhor contempla as alterações do pensamento estudadas é a de distorções do pensamento e que estas podem apresentar alta frequência nos quadros depressivos, merecendo atenção por parte dos profissionais atuantes na área de saúde mental.

\section{CONFLITO DE INTERESSE}

Os autores declaram não existir conflito de interesse. 


\section{REFERÊNCIAS}

1. Mari JJ, Jorge MR, Kohn R. Epidemiologia dos transtornos psiquiátricos em adultos. In: Mello MF, Mello AAF, Kohn R, eds. Epidemiologia da saúde mental no Brasil. Porto Alegre: Artmed; 2006, p. 119-41.

2. Keller MB, Boland RJ. Implications of failing to achieve successful long-term maintenance treatment of recurrent unipolar major depression. Biol Psychiatry. 1998;44:348-60.

3. Murray CLL, Lopez AD. The global burden of disease. A comprehensive assessment of mortality and disability from diseases, injuries and risk factors in 1990 and projected to 2020. Library of Congress Cataloging-in-Publication; 1996, p. 236-7, 262-5, 383-4, 415-7, 820-31.

4. Trichard C, Martinot JL, Alagille M. Time course of prefrontal lobe dysfunction in severely depressed in patients: a longitudinal neuropsychological study. Psychol Med. 1995;25:79-85.

5. Sougey EB, Petribu K. Depressão. In: Filgueira NA, Costa-Júnior Jl, Sá Leitão CC, Lucena VG, Melo HRL, Brito CAA. Ed. Condutas em clínica médica. 3. ed. Rio de Janeiro: Medsi; 2004. p. 599-615.

6. Sá Jr LSM. Exame mental: avaliação dos processos psicológicos afetivos. In: Sá Jr LSM, ed. Compêndio de psicopatologia e semiologia psiquiátrica, Porto Alegre: Artmed; 2001. p. 183-233.

7. Kaplan HI. Transtornos do humor. In: Kaplan HI, Sadock BJ, Grebb JA, eds. Compêndio de psiquiatria. 7. ed. Porto Alegre: Artmed; 2002, p. 493-528.

8. Beck JS. Avaliando os pensamentos automáticos, eds. Terapia cognitiva: teoria e prática. Porto Alegre: Artes Médicas; 1997, p. 116-34.

9. Calil HM, Pires MLN. Aspectos gerais das escalas de avaliação de depressão. Edição especial. Rev Psiq Clin. 1998:25(5):240-4.

10. Van Gastel A, Schotte C, Maes M. The prediction of suicidal intent in depressed patients. Acta Psychiatry Scand. 1997;96(4):254-59.

11. Vilhjalmsson R, Kristjansdottir G, Sveinbjarnardottir E. Factors associated with suicide ideation in adults. Soc Psychiatry Psychiatr Epidemiol. 1998;33:97-103.

12. Ben-Del CM, Vilela JA, Crippa JA, Hallak JE, Labate CM, Zuardi AW. Confiabilidade da "Entrevista Clínica Estruturada para o DSM-IV - Versão Clínica" traduzida para o português. Rev Bras Psiquiatr. 2001;23(3):156-9.

13. Gorestein C, Andrade L. Inventário de depressão de Beck: propriedades psicométricas da versão em português. Edição especial. Rev Psiq Clin. 1998;25(5):245-50.

14. Weissman MM, Bland RC, Canino GJ. Cross-national epidemiology of major depression and bipolar disorder. JAMA. 1996;276:293-9.

15. Kessler RC, McGonagle KA, Swartz M, Blazer DG, Nelson CB. Sex and depression in the National Comorbidity Survey. I: Lifetime prevalence, chronicity and recurrence. J Affect Disord. 1993;29(2-3):85-96.
16. Kessler RC, Berglund P, Demler O, Jin R, Koretz D, Merikangas KR, et al. The epidemiology of major depressive disorder: results from National Comorbidity Survey Replication (NCS-R). JAMA. 2003;289:3095-105.

17. Mueller TT, Leon AC, Keller MB, Solomon DA, Endicott J, Coryell W, et al. Recurrence after recovery form major depressive disorder during 15 years of observational follow-up. Am J Psychiatry. 1999;156:1000-6.

18. Botega NJ, Cano F0, Kohn SC, Knoll Al, Pereira WAB, Bonardi CM. Tentativa de suicídio e adesão ao tratamento: um estudo descritivo em hospital geral. J Bras Psiquiatr. 1995:44(1):19-25

19. Hall RCW, Platt DE. Suicide risk assessment: a review of risk factors for suicide in $100 \mathrm{pa}-$ tients who made severe suicide attempts. Evaluation of suicide risk in time of managed care. Psychosom. 1999;40(1):18-27.

20. Soares K, Almeida-Filho N, Coutinho ESF, Mari JJ. Sintomas depressivos entre os adolescentes e adultos de uma amostra populacional de três centros urbanos brasileiros: análise de dados do "Estudo Multicêntrico de Morbidade Psiquiátrica". Edição especial. Rev Psiq Clin. 1999;26(5).

21. Veras AB, Arabella R, Valença AM, Nardi AE. Prevalência de transtornos depressivos e ansiosos em uma amostra ambulatorial brasileira de mulheres na menopausa. Rev Psiquiatr RS. 2006;28(2):130-4.

22. Gazalle FK, Silva de Lima M, Tavares BF, Hallal PC. Sintomas depressivos e fatores associados em população idosa no Sul do Brasil. Rev Saude Publica. 2004;38(3):365-71.

23. Jenkins $R$, Meltzer $H$. The national survey of psychiatric morbidity in Great Britain. Soc Psychiatr Psychiatr Epidemiol. 1995;30:1-4.

24. Botega NJ, Barros MBA, Oliveira HB, Dalgallarondo P, Marín-Péon L. Suicidal behavior in the community: prevalence and factors associated with suicidal ideation. Rev Bras Psiquiatr. 2005;27(1):45-3.

25. Koenig HG, Cohen HJ, Blazer DG, Krishnan KR, Silbert TE. Profile of depressive symptoms in young and older medical inpatients with major depression. J Am Geriatr. 1993:41(11):1169-76.

26. Beck AT. The core problem in depression: the cognitive triad. Science and psychoanalysis. 1970;17:47-5.

27. Balsis S, Cully JA. Comparing depression diagnostic symptoms across younger and older adults. Aging Ment Health. 2008;12(6):800-6.

28. Spitzer RL, Williams JBW, Kroenke K, Linzer M, Hahn SR, Brody D, et al. Physical symptoms in primary care. Predictors of psychiatric disorders and functional impairment. Arch Family Med. 1994;3:774-9.

29. Ortigo KM, Westen D, Bradley B. Personality subtypes of suicide adults. J Nerv Ment Dis. 2009;197(9):687-94. 